\title{
PRODUCTS OF FLAT MODULES AND GLOBAL DIMENSION RELATIVE TO $\mathcal{F}$-MITTAG-LEFFLER MODULES
}

\author{
MANUEL CORTÉS-IZURDIAGA \\ (Communicated by Harm Derksen)
}

\begin{abstract}
Let $R$ be any ring. We prove that all direct products of flat right $R$-modules have finite flat dimension if and only if each finitely generated left ideal of $R$ has finite projective dimension relative to the class of all $\mathcal{F}$ Mittag-Leffler left $R$-modules, where $\mathcal{F}$ is the class of all flat right $R$-modules. In order to prove this theorem, we obtain a general result concerning global relative dimension. Namely, if $\mathcal{X}$ is any class of left $R$-modules closed under filtrations that contains all projective modules, then $R$ has finite left global projective dimension relative to $\mathcal{X}$ if and only if each left ideal of $R$ has finite projective dimension relative to $\mathcal{X}$. This result contains, as particular cases, the well-known results concerning the classical left global, weak and Gorenstein global dimensions.
\end{abstract}

\section{INTRODUCTION}

The motivation of this work comes from the study of right Gorenstein regular rings. A (non-necessarily commutative with unit) ring $R$ is said to be right Gorenstein regular if the category of right $R$-modules is a Gorenstein category in the sense of [9, Definition 2.18]. These rings are precisely, by [9, Theorem 2.28] and [6. Theorem 1.1], those for which the right global Gorenstein dimension is finite. Classical Iwanaga-Gorenstein rings, that is, two-sided noetherian rings with finite left and right self-injective dimensions, are left and right regular Gorenstein. Actually, the right Gorenstein regular property can be viewed as the natural one-sided generalization of the Iwanaga-Gorenstein condition to non-noetherian rings. Right Gorenstein regular rings have been studied in [9], [5], [11, [10] and [8].

In [5. Corollary VII.2.6] it is proved that a ring $R$ is right Gorenstein regular if and only if the class of all right $R$-modules with finite projective dimension coincides with the class of all right $R$-modules with finite injective dimension. A direct consequence of this fact is that the class of all modules with finite projective dimension is closed under direct products. As it is deduced from our Theorem 4.2 , rings with this property satisfy that direct products of right $R$-modules with finite flat dimension have finite flat dimension. So, in order to understand right regular

Received by the editors August 4, 2014.

2010 Mathematics Subject Classification. Primary 16D40, 16E10.

Key words and phrases. Products of flat modules, global dimension, global dimension relative to Mittag-Leffler modules.

Part of this paper was written while the author was visiting the School of Mathematics at the University of Manchester. The author is very grateful to Mike Prest for his hospitality and for many interesting discussions on the subject.

The author was partially supported by research project MTM-2014-54439 and by research group "Categorías, computación y teoría de anillos" (FQM211) of the University of Almería. 
Gorenstein rings it is necessary to study rings in which the class of right $R$-modules with finite flat dimension is closed under direct products.

The main objective of this paper is to characterize rings for which products of right modules with finite flat dimension have finite flat dimension (we shall call them left weak coherent rings, since, by the classical result [7, Theorem 2.1], they are natural extensions of left coherent rings). This is essentially done in Theorem 4.2 where we characterize left weak coherent rings as those which have finite left global dimension with respect to the $\mathcal{F}$-Mittag-Leffler modules, where $\mathcal{F}$ is the class of all flat right $R$-modules (see Definition 2.1 for details). At this point, a question arises naturally: is it possible to obtain an intrinsic description of these rings? In order to do this we study, in Section 3, the projective global dimension relative to a class, $\mathcal{X}$, consisting of left $R$-modules that contains all projective modules. The main result in this section, Corollary [3.6, states that (when $\mathcal{X}$ is closed under filtrations), the left global projective dimension relative to $\mathcal{X}$ is finite if and only if every left ideal has finite projective dimension relative of $\mathcal{X}$. Moreover, if $\mathcal{X}$ is closed under direct limits (more generally, under $\left(\aleph_{0}, \mathcal{X}\right)$-separable modules, see Definition 2.2 for details), the left global $\mathcal{X}$-projective dimension is finite if and only if each finitely generated left ideal has finite $\mathcal{X}$-projective dimension. This result is interesting on its own because it contains, as particular cases, well-known theorems concerning the left global and weak dimensions, see [3. Theorem 1] and [21, Theorem 8.25], and the left Gorenstein global dimension; see [11, Proposition $3.5]$.

This result about relative homological algebra allows us to improve the mentioned characterization of left weak coherent rings, since, in order to compute the left global dimension relative to the $\mathcal{F}$-Mittag-Leffler modules we only have to look at finitely generated left ideals. Thus, in Theorem 4.2 we characterize left weak coherent rings as those for which each finitely generated left ideal has finite $\mathcal{F}$ Mittag-Leffler dimension.

Let us point out that, as a byproduct of our results, we obtain that left coherent rings are precisely those rings with left $\mathcal{F}$-Mittag-Leffler global dimension 1: that is, rings in which submodules of $\mathcal{F}$-Mittag-Leffler left $R$-modules are again $\mathcal{F}$-MittagLeffler (see Corollary 4.3). In addition, we prove that over left coherent rings the class of all right $R$-modules with flat dimension less than or equal to $n$ (for some natural number $n$ ) is definable (see Corollary 4.8).

\section{Preliminaries}

We fix, for the rest of the paper, a non-necessarily commutative ring with identity $R$. We shall work in the categories $R$-Mod and Mod- $R$ consisting of all left and right $R$-modules respectively. Morphisms will operate on the opposite side than scalars. This implies that if $f: M \rightarrow N$ and $g: N \rightarrow L$ are morphisms in $R$-Mod, then its composition will be $f g$. We shall denote by ${ }_{R} \mathcal{F}$ the class of all flat left $R$ modules and by $\mathcal{F}_{R}$ the class of all flat right $R$-modules; we shall omit the subscript when there is no possible confusion. Given an infinite regular cardinal $\lambda$ and a left $R$-module $M$, we shall say that $M$ is $\lambda^{<}$-generated if it has a generating system with less than $\lambda$ generators. Moreover, $M$ is said to be $\lambda^{<}$-presented if there exists an exact sequence $R^{(\gamma)} \rightarrow R^{(\mu)} \rightarrow M \rightarrow 0$ with $\mu$ and $\gamma$ cardinals smaller than $\lambda$. If $\mathcal{X}$ is a class of left $R$-modules, we shall denote by $\mathcal{X}^{<\lambda}$ the class of all $\lambda^{<}$-presented 
modules belonging to $\mathcal{X}$. For any set $A,|A|$ will be its cardinality. We shall denote by $\omega$ the set of natural numbers.

A direct system of left $R$-modules, $\left(M_{i}, \tau_{i j}\right)_{i, j \in I}$, is called well-ordered if $I$ is an ordinal. Let $\kappa$ be an ordinal and $\left(M_{\alpha}, \tau_{\alpha \beta}\right)_{\alpha<\beta<\kappa}$ a well-ordered system of left $R$-modules. Following [15, Definition 3.1.1], we shall say that the direct system is continuous if for each limit ordinal $\beta<\kappa$ the direct limit of the system $\left(M_{\gamma}, \tau_{\gamma \alpha}\right)_{\gamma<\alpha<\beta}$ is the module $M_{\beta}$ with $\tau_{\alpha \beta}$ the structural morphism from $M_{\alpha}$ to $M_{\beta}$ for each $\alpha<\beta$. Let $\mathcal{X}$ be a class of left $R$-modules and suppose that Coker $\tau_{\alpha, \alpha+1} \in \mathcal{X}$ for each $\alpha+1<\kappa$. If each $\tau_{\alpha \beta}$ is a monomorphism for every pair $\alpha<\beta<\kappa$, we shall say that the direct system is an $\mathcal{X}$-continuous direct system of monomorphisms. If, moreover, each $\tau_{\alpha \beta}$ is an inclusion for every $\alpha<\beta<\kappa$, we shall say that the direct system is an $\mathcal{X}$-filtration. If $M$ is the direct limit of the filtration $\left(M_{\alpha \beta}, \tau_{\alpha \beta}\right)_{\alpha<\beta<\kappa}$, we say that $M$ is an $\mathcal{X}$-filtered module. Note that, in this case, $M=\bigcup_{\alpha<\kappa} M_{\alpha}$ for a continuous chain of submodules of $M,\left\{M_{\alpha}: \alpha<\kappa\right\}$, satisfying that $\frac{M_{\alpha+1}}{M_{\alpha}} \in \mathcal{X}$. We shall say that $\mathcal{X}$ is closed under filtrations (resp. $\mathcal{X}$-continuous limits of monomorphisms) if each $\mathcal{X}$-filtered module (resp. each limit of an $\mathcal{X}$-continuous system of monomorphisms) belongs to $\mathcal{X}$. It is easy to see that if $M$ is the limit of an $\mathcal{X}$-continuous system of monomorphisms, then it is an $\mathcal{X}$-filtered module. Then $\mathcal{X}$ is closed under an $\mathcal{X}$-continuous direct system of monomorphisms if and only if it is closed under $\mathcal{X}$-filtrations.

The class $\mathcal{X}$ is called deconstructible (see [22, Definition 1.4]) if there exists a set of modules $\mathcal{S}$ of $\mathcal{X}$ such that $\mathcal{X}$ is equal to Filt- $\mathcal{S}$, the class of all $\mathcal{S}$-filtered modules. As a consequence of [22, Lemma 1.6], $\mathcal{X}$ is deconstructible if and only if it is closed under filtrations and there exists a set $\mathcal{S}$ of $\mathcal{X}$ such that $\mathcal{X} \subseteq$ Filt- $\mathcal{S}$.

Recall the notion of Mittag-Leffler with respect to a class of modules; see 20] and [2].

Definition 2.1. Let $\mathcal{X}$ be any class of right $R$-modules. A left $R$-module $M$ is said to be $\mathcal{X}$-Mittag-Leffler if for each family of right modules belonging to $\mathcal{X}$, $\left\{F_{i}: i \in I\right\}$, the canonical map from $\left(\prod_{i \in I} F_{i}\right) \otimes M$ to $\prod_{i \in I} F_{i} \otimes M$ is monic.

For any class of right $R$-modules $\mathcal{X}$, we shall denote by $\mathcal{M}^{\mathcal{X}}$ the class consisting of all $\mathcal{X}$-Mittag-Leffler left $R$-modules. If $\mathcal{X}$ is equal to Mod- $R$, then we get the classical definition of a Mittag-Leffler module, which was introduced by Raynaud and Gruson in [19]. We are specially interested in $\mathcal{F}_{R}$-Mittag-Leffler left $R$-modules, which will be called Mittag-Leffler modules with respect to the flat modules too. Of course, there are modules which are Mittag-Leffler with respect to the flat modules but that are not Mittag-Leffler. This follows from the facts that all left $R$-modules are Mittag-Leffler if and only if $R$ is left pure-semisimple (see [4, Theorem 8]), and that all left $R$-modules are Mittag-Leffler with respect to the flats if and only if $R$ is left noetherian (this follows easily from [16, Theorem 1]). Then, if $R$ is left noetherian and not left pure-semisimple, there exist modules in $R$-Mod which are $\mathcal{F}$-Mittag-Leffler but not Mittag-Leffler.

By [17, Theorem 2.6], the Mittag-Leffler property can be characterized in terms of some local freeness. Let us recall what this local free property means.

Definition 2.2. Let $\mathcal{X}$ be a class of left $R$-modules and $\kappa$ an infinite regular cardinal. We shall say that a left $R$-module $M$ is $(\kappa, \mathcal{X})$-free if it has a $(\kappa, \mathcal{X})$-dense 
system of submodules, that is, a direct family of submodules, $\mathcal{S}$, such that:

(1) $\mathcal{S}$ is closed under well-ordered ascending chains of length smaller than $\kappa$, and

(2) every subset of $M$ of cardinality smaller than $\kappa$ is contained in an element of $\mathcal{S}$.

Given any class $\mathcal{X}$ of left $R$-modules, a module $M$ is $\left(\aleph_{0}, \mathcal{X}\right)$-free if and only if it is the union of a direct family of submodules belonging to $\mathcal{X}$. If $\mathcal{X}$ is in Mod- $R$, it is proved in [17, Theorem 2.6] that the $\mathcal{X}$-Mittag-Leffler modules are precisely the $\left(\aleph_{1}, \mathcal{M}^{\mathcal{X}}\right)$-free modules.

Finally, let us fix some notation concerning the category of short exact sequences of left $R$-modules, Ses $(R$-Mod). Recall that a morphism in this category is a triple $\left(f^{1}, f^{2}, f^{3}\right)$ making the corresponding diagrams commutative. Given three classes, $\mathcal{X}_{1}, \mathcal{X}_{2}$ and $\mathcal{X}_{3}$, of left $R$-modules, we shall denote by $\operatorname{Ses}\left(\mathcal{X}_{1}, \mathcal{X}_{2}, \mathcal{X}_{3}\right)$ the full subcategory of $\operatorname{Ses}(R$-Mod) consisting of all short exact sequences with the first term in $\mathcal{X}_{1}$, the second term in $\mathcal{X}_{2}$ and the third term in $\mathcal{X}_{3}$. We shall use continuous limits and filtrations in the category $\operatorname{Ses}(R$-Mod), which are defined in the same way as in the category of $R$-Mod.

\section{Global dimension Relative to a Class}

In this section we prove that the ring $R$ has finite left projective global dimension relative to a class $\mathcal{X}$ which is closed under filtrations and contains all projective modules if and only if each left ideal of $R$ has finite projective dimension relative to $\mathcal{X}$. As we mentioned in the introduction, this result is crucial to characterize when products of right modules with finite flat dimension have finite flat dimension.

Definition 3.1. Let $\mathcal{X}$ be a class of left $R$-modules containing all projective modules.

(1) Given a natural number $n$ and a left $R$-module $M$, we shall say that $M$ has projective dimension relative to $\mathcal{X}$ (or $\mathcal{X}$-projective dimension) less than or equal to $n$ if there exists projective resolution of $M$ such that its $(n-1) s t$ syzygy belongs to $\mathcal{X}$. We shall denote by $\mathcal{X}_{n}$ the class of all modules with $\mathcal{X}$-projective dimension less than or equal to $n$.

(2) The left global projective dimension relative to $\mathcal{X}$ (or left $\mathcal{X}$-projective global dimension) of $R$ is the supremum of the set consisting of the $\mathcal{X}$ projective dimensions of all left $R$-modules, if this supremum exists, and $\infty$ otherwise.

Let $\mathcal{X}$ be any class of left $R$-modules that contains all projective modules. It is very easy to prove that $\left(\mathcal{X}_{n}\right)_{1}=\mathcal{X}_{n+1}$ for each natural number $n$. Consequently, a left $R$-module $M$ has $\mathcal{X}$-projective dimension less than or equal to $n+1$ if and only if there exists a short exact sequence

$$
0 \longrightarrow \mathrm{K} \longrightarrow \mathrm{P} \longrightarrow \mathrm{M} \longrightarrow
$$

with $P$ projective and $K$ with $\mathcal{X}$-projective dimension less than or equal to $n$. If, in addition, $\mathcal{X}$ is closed under direct summands and finite direct sums, the $\mathcal{X}$ projective dimension does not depend on the chosen projective resolution, since, for each natural number $n$, any two $n$-sysygies of a module are projectively equivalent by [21, Proposition 8.5]. Finally, if $\mathcal{X}$ is the left hand class of a hereditary cotorsion 
pair cogenerated by a set, then, by [13, Proposition 1.11], the $\mathcal{X}$-projective dimension can be computed using exact sequences with terms in $\mathcal{X}$ or left $\mathcal{X}$-resolutions in the sense of [12, Definition 8.1.2]. Moreover, in this case, the $\mathcal{X}$-projective dimension of a module $M$ is the least natural number $n$ such that $\operatorname{Ext}_{R}^{n}(M, C)=0$ for each $C \in \mathcal{X}^{\perp}$.

Let $\mathcal{X}$ be any class of modules. In the following result we establish that some closure properties of $\mathcal{X}$ are inherited by $\mathcal{X}_{n}$. These properties will be useful to compute global dimensions.

Proposition 3.2. Let $\mathcal{X}$ be a class of left $R$-modules that contains all projective modules. Then

(1) If $\mathcal{X}$ is closed under filtrations, then $\mathcal{X}_{n}$ is closed under filtrations for each $n \in \omega$.

(2) If $\mathcal{X}$ is deconstructible, then $\mathcal{X}_{n}$ is deconstructible for each $n \in \omega$.

(3) Suppose that $\mathcal{X}$ is closed under direct summands and finite direct sums. Let $\kappa$ be any infinite regular cardinal such that each $(\kappa, \mathcal{X})$-free module belongs to $\mathcal{X}$. Then, each $\left(\kappa, \mathcal{X}_{n}\right)$-free module belongs to $\mathcal{X}_{n}$ for every $n \in \omega$.

Proof. (1) We shall induct on $n$. Case $n=0$ is true by hypothesis. Suppose that we have proven the result for some natural number $n$ and let us prove it for $n+1$. Let $M$ be an $\mathcal{X}_{n+1}$-filtered module and let $\left\{M_{\alpha}: \alpha<\kappa\right\}$ be an $\mathcal{X}_{n+1}$-filtration of $M$ for some ordinal $\kappa$. Take, for each $\alpha<\kappa$, a short exact sequence

$$
\overline{\mathbf{S}}_{\alpha+1}: \quad 0 \longrightarrow \bar{X}_{\alpha+1} \stackrel{\bar{f}_{\alpha+1}}{\longrightarrow} \bar{P}_{\alpha+1} \stackrel{\bar{g}_{\alpha+1}}{\longrightarrow} \frac{M_{\alpha+1}}{M_{\alpha}} \longrightarrow 0
$$

with $\bar{P}_{\alpha+1}$ projective and $\bar{X}_{\alpha+1} \in \mathcal{X}_{n}$. We will construct a $\operatorname{Ses}\left(\mathcal{X}_{n}, \mathcal{P}, R\right.$-Mod)continuous direct system in $\operatorname{Ses}(R$-Mod $),\left(\mathbf{S}_{\alpha}, \tau_{\alpha \beta}\right)_{\alpha \leq \beta<\kappa}$, with

$$
\mathbf{S}_{\alpha}: \quad 0 \longrightarrow X_{\alpha} \stackrel{f_{\alpha}}{\longrightarrow} P_{\alpha} \stackrel{g_{\alpha}}{\longrightarrow} M_{\alpha} \longrightarrow 0
$$

satisfying:

(i) $\tau_{\alpha, \alpha+1}^{1}: X_{\alpha} \rightarrow X_{\alpha+1}$ is a monomorphism, $\tau_{\alpha, \alpha+1}^{2}: P_{\alpha} \rightarrow P_{\alpha+1}$ is a split monomorphism and $\tau_{\alpha, \alpha+1}^{3}: M_{\alpha} \rightarrow M_{\alpha+1}$ is the inclusion.

(ii) For each $\beta<\kappa$ limit, $\mathbf{S}_{\beta}=\underset{\alpha<\beta}{\lim } \mathbf{S}_{\alpha}$.

These $S_{\alpha}$ and $\tau_{\gamma \alpha}$, for each $\gamma<\alpha<\kappa$, can be constructed recursively. Let us sketch this construction. Case $\alpha=0$ is trivial. Given any $\alpha$ such that $\mathbf{S}_{\alpha}$ and $\tau_{\gamma \alpha}$ have been already constructed for each $\gamma<\alpha$, we can construct, as in the proof of the Horseshoe lemma, 21, Proposition 6.24], a commutative diagram with exact 
rows and columns

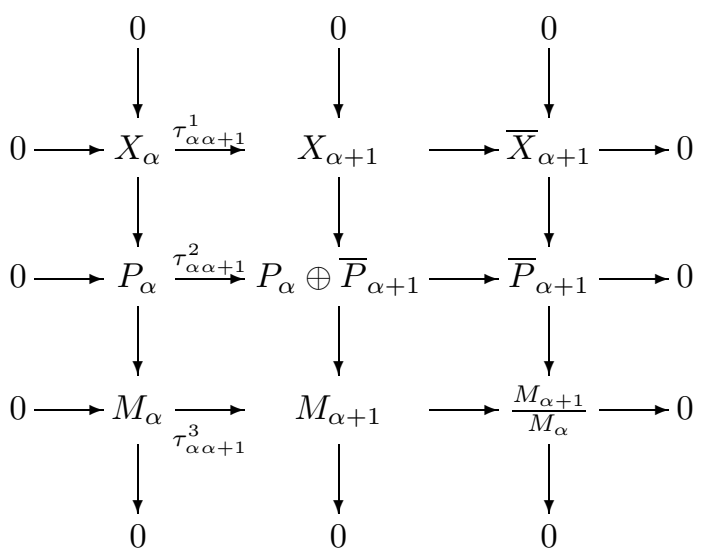

Then take $\mathcal{S}_{\alpha+1}$ to be the middle column of the diagram with $P_{\alpha+1}=P_{\alpha} \oplus \bar{P}_{\alpha+1}$. If $\alpha$ is a limit ordinal and we have already constructed the sequence $\mathbf{S}_{\gamma}$ for each $\gamma<\alpha$, then take $\mathbf{S}_{\alpha}$ to be the direct limit of the system $\left(\mathbf{S}_{\gamma}, \tau_{\gamma \beta}\right)_{\gamma \leq \beta<\alpha}$. This finishes the construction.

Now let

$$
\mathbf{S}: 0 \longrightarrow X \longrightarrow P \longrightarrow N
$$

be the direct limit of the system $\left(\mathbf{S}_{\alpha}, \tau_{\alpha \beta}\right)_{\alpha<\beta<\kappa}$. Since direct limits in the category of short exact sequences are computed componentwise, we get that $N=M$. Moreover, we conclude that $X$ is an $\mathcal{X}_{n}$-filtered module and, by induction hypothesis, it belongs to $\mathcal{X}_{n}$; and that $P$, being filtered by projective modules, is projective. Then the short exact sequence $\mathbf{S}$ says that $M \in \mathcal{X}_{n+1}$ and the proof is finished.

(2) If $\mathcal{X}$ is deconstructible, then $\mathcal{X}_{n}$ is closed under $\mathcal{X}_{n}$-filtrations by (1). Moreover, if there exists a set $\mathcal{S}$ such that each module in $\mathcal{X}$ is $\mathcal{S}$-filtered, then, actually there exists an infinite regular cardinal number $\lambda$ such that $\mathcal{X} \subseteq$ Filt- $\mathcal{X}^{<\lambda}$. Then, the same proof of [13, Theorem 2.2] gives that $\mathcal{X}_{n} \subseteq$ Filt- $\mathcal{X}_{n}^{<\lambda}$ (the mentioned result is proved when $\mathcal{X}$ is the left hand class of a hereditary cotorsion pair cogenerated by a set; but in the proof it is only used that $\mathcal{X} \subseteq$ Filt- $\mathcal{X}^{<\lambda}$ for some infinite regular cardinal).

(3) In some part of the following proof we have to assume that $R$ has more than two elements. Note that if $R$ has two elements there is nothing to prove as $\mathcal{X}_{n}=\mathcal{X}$ for each $n \in \omega$.

We shall induct on $n$. Case $n=0$ is the hypothesis. Suppose that the result is true for any natural number $n$ and let us prove it for $n+1$. Let $M$ be any $\left(\kappa, \mathcal{X}_{n+1}\right)$-free module and fix $\mathcal{S}$ a $\left(\kappa, \mathcal{X}_{n+1}\right)$-dense system of $M$. For each $m \in M$ let $\varphi_{m}: R \rightarrow R m$ be the morphism given by multiplication; for each $T \leq M$, denote by $\varphi_{T}: R^{(T)} \rightarrow M$ the induced morphism and by $K_{T}$ its kernel. Let us denote by $e_{m}$ the canonical element of $R^{(M)}$ for each $m \in M$. We claim that $\mathcal{T}=\left\{K_{S}: S \in \mathcal{S}\right\}$ is a $\left(\kappa, \mathcal{X}_{n}\right)$-dense system of $K_{M}$. First of all note that, since the $\mathcal{X}$-dimension does not depend on the chosen projective resolution (as $\mathcal{X}$ is closed under direct summands and finite direct sums), $K_{S} \in \mathcal{X}_{n}$ for each $S \in \mathcal{S}$. Moreover, $\mathcal{T}$ is trivially a direct system and each $X \leq K_{M}$ with $|X|<\kappa$ is contained in some $K_{S}$ for some $S \in \mathcal{S}$. 
It only remains to prove that $\mathcal{T}$ is closed under well-ordered ascending chains of length smaller than $\kappa$. First of all, let us prove that if $K_{S} \leq K_{T}$ for some $S, T \in \mathcal{S}$, then $S \leq T$. Let $m \in S$ and suppose that $m \notin T$ and take $r \in R-\{1\}$. Since $r e_{m}-e_{r m}$ and $(1-r) e_{m}-e_{(1-r) m}$ are non-zero elements in $K_{S} \leq K_{T}$ and $m \notin T$, we conclude that both $r m$ and $(1-r) m$ belong to $T$ (note that, if $L \in \mathcal{S}$ and $l, t \notin L$, then $K_{L} \cap\left(R_{l} \oplus R_{t}\right)=0, R_{l}$ and $R_{t}$ being the copies of $R$ in coordinates $l$ and $s$ respectively). Then $m=r m+(1-r) m$ belongs to $T$, a contradiction. Now, using this fact, each well-ordered chain $\left\{K_{S_{\alpha}}: \alpha<\mu\right\}$ of modules in $\mathcal{T}$ (with $\mu<\kappa)$, gives, taking unions, the short exact sequence

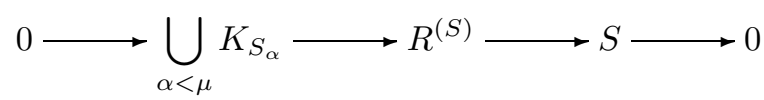

where $S=\bigcup_{\alpha<\mu} S_{\alpha}$. Then, since $\bigcup_{\alpha<\mu} K_{S_{\alpha}}=K_{S}$ and $S \in \mathcal{S}$, we conclude that $\bigcup_{\alpha<\mu} K_{S_{\alpha}} \in \mathcal{T}$. Consequently, $\mathcal{T}$ is closed under well-ordered unions.

The conclusion is that $K_{M}$ is $\left(\kappa, \mathcal{X}_{n}\right)$-free and by induction hypothesis, $K_{M} \in \mathcal{X}_{n}$. Consequently, $M \in \mathcal{X}_{n+1}$ and the proof is finished.

Remark 3.3. (1) of Proposition 3.2 extends [13, Lemma 2.1], where it is proved that $\mathcal{X}_{n}$ is closed under filtrations when $\mathcal{X}$ is the left hand class of a hereditary cotorsion pair cogenerated by a set.

The preceding proposition contains well-known results concerning the projective, flat and Gorenstein projective dimensions. Moreover, we can extend the characterization of Mittag-Leffler modules given in [17, Theorem 2.6] to modules with finite dimension with respect to Mittag-Leffler modules.

Corollary 3.4. Let $\mathcal{X}$ be any class of right $R$-modules, $n$ a natural number and $M$ a module. The following assertions are equivalent:

(1) $M$ has $\mathcal{M}^{\mathcal{X}}$-Mittag-Leffler dimension less than or equal to $n$.

(2) $M$ is $\left(\aleph_{1}, \mathcal{M}_{n}^{\mathcal{X}}\right)$-free.

Now we can prove the main theorem of this section:

Theorem 3.5. Let $\lambda$ be an infinite regular cardinal, $n$ a non-zero natural number and $\mathcal{X}$ a class of left $R$-modules closed under filtrations that contains all projective modules. The following assertions are equivalent:

(1) Each $\lambda$-presented module has $\mathcal{X}$-projective dimension less than or equal to $n$.

(2) Each $\lambda^{<}$-generated left ideal of $R$ has $\mathcal{X}$-projective dimension less than or equal to $n-1$.

Proof. (1) $\Rightarrow(2)$ If $I$ is a left ideal of $R$ which is $\lambda^{<}$-generated, the module $\frac{R}{I}$ is $\lambda^{<}$-presented and, by hypothesis, has $\mathcal{X}$-projective dimension less than or equal to $n$. This means that $I$ has $\mathcal{X}$-projective dimension less than or equal to $n-1$.

$(2) \Rightarrow(1)$. Let $M$ be any $\lambda^{<}$-presented module, choose $\mu$ a cardinal smaller than $\lambda$ such that there exists an epimophism $f: R^{(\mu)} \rightarrow M$ and that its kernel $K$ is $\lambda^{<}$-generated. We claim that $K$ has $\mathcal{X}$-projective dimension less than or equal to $n-1$.

In order to prove the claim, denote by $p_{\alpha}: R^{(\mu)} \rightarrow R$ and $i_{\alpha}: R \rightarrow R^{(\mu)}$ the projection and inclusion for each $\alpha<\mu$, and by $K_{\alpha}=K \cap R^{(\alpha)}$. Note that the 
family $\left\{K_{\alpha}: \alpha<\kappa\right\}$ is a filtration of $K$. Now, for each $\alpha<\kappa$, we have the exact sequence

$$
0 \longrightarrow K_{\alpha} \longrightarrow K_{\alpha+1} \stackrel{q_{\alpha}}{\longrightarrow} R
$$

where $q_{\alpha}$ is the restriction of $p_{\alpha}$ to $K_{\alpha+1}$. Note that $\operatorname{Im} q_{\alpha}$ is $\lambda^{<}$-generated: if $\left\{x_{\delta}\right.$ : $\delta<\beta\}$ is a generating set of $K$ with $\beta$ a cardinal smaller than $\lambda$, then $\left\{\left(x_{\delta}\right) p_{\gamma} i_{\gamma}\right.$ : $\delta<\beta, \gamma<\mu\}$ is a generating set of $K$, from which follows that $\left\{\left(x_{\delta}\right) p_{\gamma} i_{\gamma}: \delta<\right.$ $\beta, \gamma<\alpha+1\}$ is a generating set of $K_{\alpha+1}$ with cardinality smaller than $\lambda$. The conclusion is that $\frac{K_{\alpha+1}}{K_{\alpha}}$ is isomorphic to a $\lambda^{<}$-generated left ideal of $R$ and, by hypothesis, has $\mathcal{X}$-projective dimension less than or equal to $n-1$. This means that $K$ is $\mathcal{X}_{n-1}$-filtered and by Proposition 3.2 belongs to $\mathcal{X}_{n-1}$. This proves our claim and finishes the proof of the theorem.

Note that there always exists an infinite regular cardinal $\lambda$ such that $R$ is left $\lambda$-noetherian, in the sense that each left ideal is $\lambda^{<}$-generated. Then, as an immediate consequence of the previous result, if each $\lambda^{<}$-presented module has finite $\mathcal{X}$-projective dimension, then $R$ has finite left global $\mathcal{X}$-projective dimension. Moreover, if we do not establish any cardinal restriction, we obtain an intrinsic description of rings with finite global projective relative dimension:

Corollary 3.6. Let $n$ be a non-zero natural number and $\mathcal{X}$ a class of left $R$ modules closed under filtrations that contains all projective modules. The following assertions are equivalent:

(1) $R$ has left global $\mathcal{X}$-projective dimension less than or equal to $n$.

(2) Each left ideal of $R$ has $\mathcal{X}$-projective dimension less than or equal to $n-1$.

If, in addition, $\mathcal{X}$ is closed under direct summands, finite direct sums and each $(\kappa, \mathcal{X})$-free module belongs to $\mathcal{X}$, for some infinite regular cardinal $\kappa$, then these conditions are equivalent to:

(3) Each $\kappa^{<}$-generated left ideal of $R$ has $\mathcal{X}$-projective dimension less than or equal to $n-1$.

Proof. (1) $\Leftrightarrow$ (2) follows from the preceding theorem. (2) $\Rightarrow(3)$ is clear. In order to prove $(3) \Rightarrow(2)$ simply note that, if $I$ is a left ideal, the hypothesis says that the set of all $\kappa^{<}$-generated left ideals contained in $I$ is a $\left(\kappa, \mathcal{X}_{n-1}\right)$-dense system of $I$. By Proposition [3.2, $I$ has $\mathcal{X}$-projective dimension less than or equal to $n-1$.

This result contains, as particular cases, the well-known results concerning the global, weak and Gorenstein global dimensions (see [21, Theorem 8.16], 21, Theorem 8.25] and [11, Proposition 3.5]). Moreover, it can be applied to the $\mathcal{F}$-MittagLeffler dimension.

Corollary 3.7. Let $n$ be a non-zero natural number. Then $R$ has left $\mathcal{M}^{\mathcal{F}}$ projective global dimension less than or equal to $n$ if and only if each finitely generated left ideal has $\mathcal{M}^{\mathcal{F}}$-projective dimension less than or equal to $n-1$.

Proof. Follows from the preceding result using that the class $\mathcal{M}^{\mathcal{F}}$ is closed under filtrations by [2, Proposition 1.9] and under $\left(\aleph_{0}, \mathcal{M}^{\mathcal{F}}\right)$-free modules, that is, under direct unions of submodules, by [16, Theorem 1].

Another application of our result is when the left global and weak dimensions coincide (for example, when the ring is left noetherian or left perfect). In this case, the left global dimension is the supremum of the projective dimensions of the finitely generated left ideals. 
Corollary 3.8. Let $R$ be a ring such that the left global and weak dimensions coincide, and let $n$ be a non-zero natural number. Then $R$ has left global dimension less than or equal to $n$ if and only if each finitely generated left ideal has projective dimension less than or equal to $n-1$.

Proof. Simply note that by hypothesis and Corollary [3.6, the left global dimension is equal to the supremum of the flat dimensions of all finitely generated left ideals. But this supremum is smaller than or equal to the supremum of the projective dimension of all finitely generated left ideals.

Let us finish this section with a useful result in order to compute global dimensions. It is proven with the argument used in [5, Corollary VII.2.6].

Lemma 3.9. Let $\mathcal{X}$ and $\mathcal{Y}$ be classes of left $R$-modules such that $\mathcal{X}$ is closed under direct summands, finite direct sums and contains all projective modules, and $\mathcal{Y}$ is closed under countable direct sums or countable direct products. Then the following assertions are equivalent:

(1) Each module in $\mathcal{Y}$ has finite $\mathcal{X}$-projective dimension.

(2) There exists a natural number n such that each module in $\mathcal{Y}$ has $\mathcal{X}$-projective dimension less than or equal to $n$.

Proof. We only have to prove that (1) implies (2). We do it assuming that $\mathcal{Y}$ is closed under countable direct sums (the case of products is analogous). Suppose that (2) is false and take, for each natural number $n$, a module $Y_{n}$ in $\mathcal{Y}$ with $\mathcal{X}$ projective dimension equal to $n$. Then, $Y=\bigoplus_{n \in \omega} Y_{n}$ belongs to $\mathcal{Y}$ and has infinite $\mathcal{X}$-dimension since, otherwise, it would exist $m \in \omega$ such that $Y$ would have $\mathcal{X}$ projective dimension less than or equal to $m$, which would imply that $Y_{m+1}$ would have this property too (note that if $\mathcal{X}$ is closed under direct summands, then so is $\left.\mathcal{X}_{m}\right)$. But the dimension of $Y_{m+1}$ is $m+1$, which is a contradiction.

\section{When DiRect PRoducts of Flat modules haVe Finite Flat Dimension}

In this section we investigate rings for which direct products of right $R$-modules with finite flat dimension have finite flat dimension. As a consequence of our first result, we only have to look at a direct product of flat modules.

Proposition 4.1. The following assertions are equivalent:

(1) The direct product of any family of flat right $R$-modules has finite flat dimension.

(2) There exists a natural number $n$ such that the product of any family of flat right $R$-modules has flat dimension less than or equal to $n$.

(3) There exists a natural number $m$ such that the direct product of any family of right $R$-modules with flat dimension less than or equal to $m$ has finite flat dimension.

(4) There exist natural numbers $m$ and $n$ such that the direct product of any family of right $R$-modules with flat dimension less than or equal to $m$ has flat dimension less than or equal to $m+n$.

(5) For any natural number $m$, the direct product of any family of right $R$ modules with flat dimension less than or equal to $m$ has finite flat dimension. 
(6) For any natural number $m$ there exists a natural number $n$ such that the direct product of any family of right $R$-modules with flat dimension less than or equal to $m$ has flat dimension less than or equal to $m+n$.

Proof. (1) $\Leftrightarrow(2)$. Follows from Lemma 3.9 by taking $\mathcal{Y}$ the class of all products of flat right $R$-modules and $\mathcal{X}$ the class of all flat right $R$-modules.

$(3) \Leftrightarrow(4)$ and $(5) \Leftrightarrow(6)$. Again follows from Lemma 3.9 by taking $\mathcal{Y}$ the class consisting of all products of right $R$-modules with flat dimension less than or equal to $m$ and $\mathcal{X}$ the class of all flat right $R$-modules.

$(1) \Rightarrow(5)$. By induction on $m$. Case $m=0$ is (1). Suppose that the result is true for some natural number $m$ and let us prove it for $m+1$. Let $\left\{F_{i}: i \in I\right\}$ be a family of right $R$-modules with flat dimension less than or equal to $m+1$. Then, for each $i \in I$ there exists a projective presentation

$$
0 \longrightarrow K_{i} \longrightarrow P_{i} \longrightarrow F_{i} \longrightarrow 0
$$

with $K_{i}$ having flat dimension less than or equal to $n$. With all these sequences, we can form the short exact sequence

$$
0 \longrightarrow \prod_{i \in I} K_{i} \longrightarrow \prod_{i \in I} P_{i} \longrightarrow \prod_{i \in I} F_{i} \longrightarrow 0 \text {. }
$$

Now apply the induction hypothesis to get that $\prod_{i \in I} P_{i}$ and $\prod_{i \in I} K_{i}$ have finite flat dimension and, consequently, that $\prod_{i \in I} F_{i}$ has finite flat dimension too.

$(5) \Rightarrow(3) \Rightarrow(1)$. Trivial.

We shall say that $R$ is a left weak coherent ring if it satisfies the equivalent conditions of the preceding result. Suppose that $R$ is such a ring. Then we can take $n$ the maximum of the set consisting of all flat dimensions of all direct products of flat right $R$-modules. In this case, we shall say that $R$ is left weak $n$-coherent. Note that left weak 0 -coherent rings are precisely left coherent rings. In the following result we characterize left weak coherent rings in terms of the left global $\mathcal{M}^{\mathcal{F}}$ projective resolution.

Theorem 4.2. Let $n$ be a natural number. The following assertions are equivalent:

(1) $R$ is left weak $n$-coherent.

(2) There exists a set $I_{0}$ with $\left|I_{0}\right| \geq|R|$ such that $R_{R}^{I_{0}}$ has flat dimension less than or equal to $n$.

(3) $R$ has left global $\mathcal{M}^{\mathcal{F}}$-projective dimension less than or equal to $n+1$.

(4) Each finitely generated left ideal of $R$ has $\mathcal{M}^{\mathcal{F}}$-projective dimension less than or equal to $n$.

(5) Each cyclic left $R$-modules has $\mathcal{M}^{\mathcal{F}}$-projective dimension less than or equal to $n+1$.

Proof. (1) $\Rightarrow(2)$. Trivial.

$(2) \Leftrightarrow(3)$. Let $M$ be a left $R$-module. We claim that the $\mathcal{M}^{\mathcal{F}}$-projective dimension of $M$ is less than or equal to $n$ if and only if $\operatorname{Tor}_{n}^{R}\left(R^{I_{0}}, M\right)=0$. Then the equivalence of (2) and (3) follows from this claim.

We proceed by induction on $n$. Fix a projective presentation of $M$,

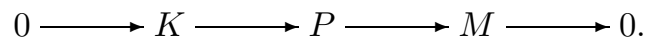


In order to prove case $n=1$ consider the following commutative diagram with exact rows:

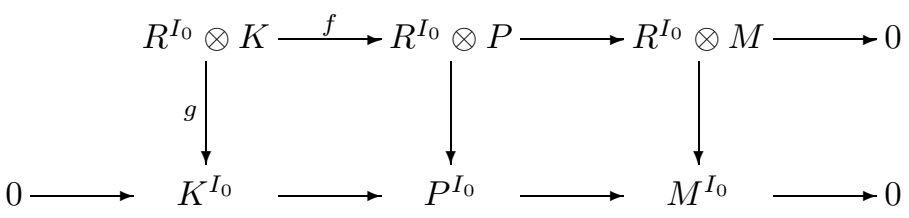

Since $\operatorname{Tor}_{1}^{R}\left(R^{I_{0}}, P\right)=0$, as $P$ is projective, we conclude that $\operatorname{Tor}_{1}^{R}\left(R^{I_{0}}, N\right)=0$ if and only if $f$ is monic, if and only if $g$ is monic if and only if $K$ is $\mathcal{F}$-Mittag-Leffler by [16, Theorem 1], if and only if $M$ has $\mathcal{M}^{\mathcal{F}}$-projective dimension less than or equal to 1.

Now suppose that we have proved the claim for some non-zero natural number $n$. Then, looking at the exact sequence (4.1), we get that $\operatorname{Tor}_{n+1}^{R}\left(R^{I_{0}}, M\right)=0$ if and only if $\operatorname{Tor}_{n}^{R}\left(R^{I_{0}}, K\right)=0$, if and only if the $\mathcal{M}_{\mathcal{F}}$-projective dimension of $K$ is less than or equal to $n$ (by induction hypothesis), if and only if the $\mathcal{M}_{\mathcal{F}}$-projective dimension of $M$ is less than or equal to $n+1$.

$(3) \Leftrightarrow(4) \Leftrightarrow(5)$. These are Corollary 3.7 .

When $n=0$ we obtain new characterizations of left coherent rings:

Corollary 4.3. The following assertions are equivalent:

(1) $R$ is left coherent.

(2) For each natural number $m$, the direct product of any family of right $R$ modules with flat dimension less than or equal to $m$ has flat dimension less than or equal to $m$.

(3) Each left $R$-module has $\mathcal{M}^{\mathcal{F}}$-projective dimension less than or equal to 1.

(4) Each left ideal of $R$ is $\mathcal{F}$-Mittag-Leffler.

(5) Each submodule of an $\mathcal{F}$-Mittag Leffler left $R$-module is $\mathcal{F}$-Mittag Leffler.

Proof. (1) $\Leftrightarrow(2)$. If $R$ is left coherent, direct products of flat right $R$-modules are flat by Chase's result, [7, Theorem 2.1]. Then, the same proof as (1) $\Rightarrow(3)$ in Proposition 4.1 gives the equivalence of (1) and (2).

The equivalences of (1), (3) and (4) follow from the previous theorem. (5) $\Rightarrow(3)$ is clear. It remains to prove that (3) implies (5). Let $M$ be an $\mathcal{F}$-Mittag-Leffler left $R$-module and $K \leq M$ a submodule. Take a projective presentation $f: P \rightarrow \frac{M}{K}$, and, making pullback, we can construct the following commutative diagram with exact rows and columns:

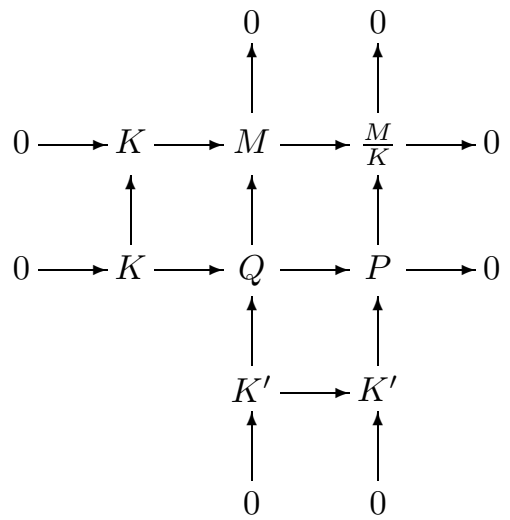


By (3), $K^{\prime}$ belongs to $\mathcal{M}^{\mathcal{F}}$ and by [20, Corollary 2.4], $Q$ belongs to $\mathcal{M}_{\mathcal{F}}$ too. Since the middle row is split exact, $K$ belongs to $\mathcal{M}^{\mathcal{F}}$ again by [20, Corollary 2.4] and the proof is finished.

This result says that rings in which the class $\mathcal{M}^{\mathcal{F}}$ is closed under submodules, that is, rings with left global $\mathcal{M}^{\mathcal{F}}$-projective dimension equal to 1 are, precisely, left coherent rings. Recall that, as it can be easily deduced from [16. Theorem 1], rings with left global $\mathcal{M}^{\mathcal{F}}$-projective dimension zero are left noetherian rings.

Examples 4.4. (1) There exist left weak coherent rings which are not left coherent. For example, let $R$ be a non-semihereditary ring with weak dimension 1 (such a ring is constructed in [14, Example 3.1.2]). Then $R$ is weak 1-coherent, but it is not left coherent by [14, Theorem 3.1.3].

(2) There exist left weak coherent rings which have infinite weak global dimension. For instance, in [18, Example 7.7.2] it is given a noetherian ring which has infinite global dimension. Since, for noetherian rings, the global and weak dimensions coincide, this ring has infinite weak dimension too.

(3) Right regular Gorenstein rings are left weak coherent. This is because, as it has been proved in [5, Corollary VII.2.6], for these rings the class of all right $R$-modules with finite projective dimension coincides with the class of all modules with finite injective dimension. In particular, this means that the class of all right $R$-modules with finite projective dimension is closed under direct products. Then all direct products of copies of $R_{R}$ have finite flat dimension. By Theorem 4.2, $R$ is left weak coherent.

Let us finish the paper with some applications of our results. In the first of them we characterize when the class of all right $R$-modules with finite flat dimension is closed under products. Recall that the right finitistic flat dimension is the supremum of the set consisting of the flat dimensions of all right $R$-modules with finite flat dimension, if this supremum exists, and $\infty$ otherwise.

Corollary 4.5. The following assertions are equivalent:

(1) The class of all right $R$-modules with finite flat dimension is closed under direct products.

(2) $R$ is left weak coherent and has finite right finitistic flat dimension.

Proof. $(1) \Rightarrow(2)$. By Lemma 3.9 taking $\mathcal{X}$ and $\mathcal{Y}$ the class of all flat modules.

$(2) \Rightarrow(1)$. Trivial.

Recall that a class of right $R$-modules is definable if it is closed under products, pure submodules and direct limits. As a second application of our results, we are going to prove that the class of all right $R$-modules with flat dimension less than or equal to $n$ is definable for every $n \in \omega$, when $R$ is a left coherent ring. We shall use the following preliminary lemma that says that, in order to see that a class is closed under direct limits, we only have to prove that it is closed under continuous well-ordered limits. The proof can be done with the same argument used in [1, Corollary 1.7].

Lemma 4.6. Let $\mathcal{X}$ be a class of right $R$-modules which is closed under continuous well-ordered limits. Then $\mathcal{X}$ is closed under direct limits. 
Proposition 4.7. For each natural number $n$, the class of all right $R$-modules with flat dimension less than or equal to $n$ is closed under direct limits and pure submodules.

Proof. First of all, we shall prove that $\mathcal{F}_{n}$ is closed under direct limits. In view of the preceding result, we only have to see that $\mathcal{F}_{n}$ is closed under continuous well-ordered direct limits. We shall make the proof inductively on $n$. Case $n=0$ is the well-known property of flat modules. Assume that we have proved the result for some natural number $n$ and let us prove it for $n+1$. Let $\left(M_{\alpha}, \tau_{\alpha \beta}\right)_{\alpha<\beta<\kappa}$ be a continuous well-ordered system of modules belonging to $\mathcal{F}_{n+1}$. We are going to construct, for each $\alpha<\kappa$, a direct system in $\operatorname{Ses}\left(\mathcal{F}_{n}, \mathcal{F}, \operatorname{Mod}-R\right)$,

$$
\left(0 \longrightarrow F_{\gamma} \stackrel{f_{\gamma}}{\longrightarrow} G_{\gamma} \stackrel{g_{\gamma}}{\longrightarrow} M_{\gamma} \longrightarrow 0,\left(\tau_{\delta \gamma}^{1}, \tau_{\delta, \gamma}^{2}, \tau_{\delta \gamma}^{3}\right)\right)_{\delta<\gamma \leq \alpha}
$$

with $\tau_{\gamma \alpha}^{3}=\tau_{\gamma \alpha}$ for each $\gamma \leq \alpha$. Then, the result will follow by taking direct limits, since the resulting exact sequence belongs to $\operatorname{Ses}\left(\mathcal{F}_{n}, \mathcal{F}, \operatorname{Mod}-R\right)$ and its last term is $M$ (note that flat dimension can be computed using flat resolutions).

We shall make the construction by transfinite recursion on $\alpha$. If $\alpha=0$ simply take a short exact sequence

$$
0 \longrightarrow F_{0} \stackrel{f_{0}}{\longrightarrow} G_{0} \stackrel{g_{0}}{\longrightarrow} M_{0} \longrightarrow 0
$$

with $G_{0}$ flat and $F_{0} \in \mathcal{F}_{n}$. Now fix $\alpha>0$ and assume that we have made the construction for each ordinal smaller than $\alpha$. If $\alpha$ is successor, say $\alpha=\gamma+1$, take a short exact sequence

$$
0 \longrightarrow F_{\alpha} \stackrel{f_{\alpha}}{\longrightarrow} G_{\alpha} \stackrel{g_{\alpha}}{\longrightarrow} M_{\alpha} \longrightarrow 0
$$

with $g_{\alpha}$ a flat precover of $M_{\alpha}$. Then $F_{\alpha}$ has flat dimension less than or equal to $n$. Now, using the factorization property of the precover, we can construct the commutative diagram,

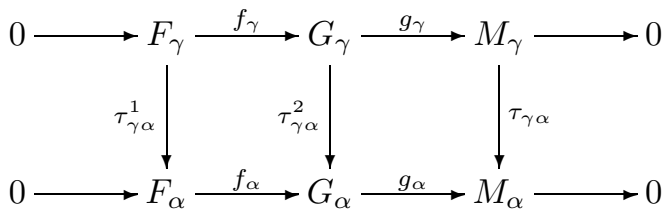

Then if we set $\tau_{\gamma \alpha}^{3}=\tau_{\gamma \alpha}$ and $\tau_{\delta \alpha}^{i}=\tau_{\gamma \alpha}^{i} \tau_{\delta \gamma}^{i}$ for each $i \in\{1,2,3\}$ and $\delta<\gamma$, we will have the successor case done. If $\alpha$ is limit the construction is made by taking direct limits.

Now we prove that $\mathcal{F}_{n}$ is closed under pure submodules. Take a pure short exact sequence

$$
0 \longrightarrow \mathrm{K} \longrightarrow \mathrm{M} \longrightarrow \mathrm{\longrightarrow} \longrightarrow 0
$$

with $M \in \mathcal{F}_{n}$. Then there exists a direct system of splitting exact sequences,

$$
\left(0 \longrightarrow K_{i} \stackrel{f_{i}}{\longrightarrow} M_{i} \stackrel{g_{i}}{\longrightarrow} L_{i} \longrightarrow 0,\left(\tau_{i j}^{1}, \tau_{i j}^{2}, \tau_{i j}^{3}\right)\right)_{i<i \in I},
$$

whose direct limit is (4.2). For any module $C$, if we apply $\operatorname{Tor}_{n+1}^{R}(-, C)$ to this system, we get another direct system of splitting short exact sequences whose limit is

$$
0 \longrightarrow \lim _{\longrightarrow} \operatorname{Tor}_{n+1}^{R}\left(K_{i}, C\right) \longrightarrow \lim _{\longrightarrow} \operatorname{Tor}_{n+1}^{R}\left(M_{i}, C\right) \longrightarrow \lim _{\longrightarrow} \operatorname{Tor}_{n+1}^{R}\left(L_{i}, C\right) \longrightarrow 0 .
$$


Now, using that $\operatorname{Tor}_{n+1}^{R}$ commutes with direct limits (see, for example, [21, Proposition 7.8$]$ ), we actually get the short exact sequence

$$
0 \longrightarrow \operatorname{Tor}_{n+1}^{R}(K, C) \longrightarrow \operatorname{Tor}_{n+1}^{R}(M, C) \longrightarrow \operatorname{Tor}_{n+1}^{R}(L, C) \longrightarrow
$$

Finally, since $M$ has flat dimension less than or equal to $n$, we conclude that $\operatorname{Tor}_{n+1}^{R}(M, C)=0$ and, consequently, $\operatorname{Tor}_{n+1}^{R}(K, C)=0$. Since $C$ was arbitrary, $K$ has flat dimension less than or equal to $n$ and the proof is finished.

Note that as a consequence of [1, Corollary 2.36], this result says that, for any ring and $n \in \omega$, the full subcategory of Mod- $R$ whose class of objects consists of all modules with flat dimension less than or equal to $n$ is an accessible category.

Corollary 4.8. Let $n$ be a natural number and suppose that $R$ is left coherent. Then the class of all right $R$-modules with flat dimension less than or equal to $n$ is definable.

Proof. If $R$ is left coherent, then the class $\mathcal{F}_{n}$ is closed under direct products (this can be deduced with the same argument used in Proposition 4.1). Then the result follows from the previous proposition.

\section{REFERENCES}

[1] Jiří Adámek and Jiří Rosický, Locally presentable and accessible categories, London Mathematical Society Lecture Note Series, vol. 189, Cambridge University Press, Cambridge, 1994. MR.1294136

[2] Lidia Angeleri Hügel and Dolors Herbera, Mittag-Leffler conditions on modules, Indiana Univ. Math. J. 57 (2008), no. 5, 2459-2517, DOI 10.1512/iumj.2008.57.3325. MR2463975

[3] Maurice Auslander, On the dimension of modules and algebras. III. Global dimension, Nagoya Math. J. 9 (1955), 67-77. MR.0074406

[4] Goro Azumaya and Alberto Facchini, Rings of pure global dimension zero and Mittag-Leffler modules, J. Pure Appl. Algebra 62 (1989), no. 2, 109-122, DOI 10.1016/0022-4049(89)901461. MR1027751

[5] Apostolos Beligiannis and Idun Reiten, Homological and homotopical aspects of torsion theories, Mem. Amer. Math. Soc. 188 (2007), no. 883, viii+207, DOI 10.1090/memo/0883. MR2327478

[6] Driss Bennis and Najib Mahdou, Global Gorenstein dimensions, Proc. Amer. Math. Soc. 138 (2010), no. 2, 461-465, DOI 10.1090/S0002-9939-09-10099-0. MR2557164

[7] Stephen U. Chase, Direct products of modules, Trans. Amer. Math. Soc. 97 (1960), 457-473. MR0120260

[8] Edgar E. Enochs, Manuel Cortés-Izurdiaga, and Blas Torrecillas, Gorenstein conditions over triangular matrix rings, J. Pure Appl. Algebra 218 (2014), no. 8, 1544-1554, DOI 10.1016/j.jpaa.2013.12.006. MR3175039

[9] E. Enochs, S. Estrada, and J. R. García-Rozas, Gorenstein categories and Tate cohomology on projective schemes, Math. Nachr. 281 (2008), no. 4, 525-540, DOI 10.1002/mana.200510622. MR2404296

[10] E. Enochs, S. Estrada, and A. Iacob, Rings with finite Gorenstein global dimension, Math. Scand. 102 (2008), no. 1, 45-58. MR2420678

[11] Edgar E. Enochs, Alina Iacob, and Overtoun M. G. Jenda, Closure under transfinite extensions, Illinois J. Math. 51 (2007), no. 2, 561-569. MR.2342674

[12] Edgar E. Enochs and Overtoun M. G. Jenda, Relative homological algebra. Volume 1, Second revised and extended edition, de Gruyter Expositions in Mathematics, vol. 30, Walter de Gruyter GmbH \& Co. KG, Berlin, 2011. MR2857612

[13] M. Cortés Izurdiaga, S. Estrada, and P. A. Guil Asensio, A model structure approach to the finitistic dimension conjectures, Math. Nachr. 285 (2012), no. 7, 821-833, DOI 10.1002/mana.201000116. MR2924515 
[14] Sarah Glaz, Prüfer conditions in rings with zero-divisors, Arithmetical properties of commutative rings and monoids, Lect. Notes Pure Appl. Math., vol. 241, Chapman \& Hall/CRC, Boca Raton, FL, 2005, pp. 272-281, DOI 10.1201/9781420028249.ch17. MR2140700

[15] Rüdiger Göbel and Jan Trlifaj, Approximations and endomorphism algebras of modules, de Gruyter Expositions in Mathematics, vol. 41, Walter de Gruyter GmbH \& Co. KG, Berlin, 2006. MR2251271

[16] K. R. Goodearl, Distributing tensor product over direct product, Pacific J. Math. 43 (1972), 107-110. MR0311714

[17] Dolors Herbera and Jan Trlifaj, Almost free modules and Mittag-Leffler conditions, Adv. Math. 229 (2012), no. 6, 3436-3467, DOI 10.1016/j.aim.2012.02.013. MR2900444

[18] J. C. McConnell and J. C. Robson, Noncommutative Noetherian rings, Revised edition, Graduate Studies in Mathematics, vol. 30, American Mathematical Society, Providence, RI, 2001. With the cooperation of L. W. Small. MR1811901

[19] Michel Raynaud and Laurent Gruson, Critères de platitude et de projectivité. Techniques de "platification" d'un module (French), Invent. Math. 13 (1971), 1-89. MR0308104

[20] Ph. Rothmaler, Mittag-Leffler Modules and Positive Atomicity, Habilitantionsschrift, Kiel, 1994.

[21] Joseph J. Rotman, An introduction to homological algebra, 2nd ed., Universitext, Springer, New York, 2009. MR2455920

[22] Jan Št'ovíček, Deconstructibility and the Hill lemma in Grothendieck categories, Forum Math. 25 (2013), no. 1, 193-219. MR.3010854

Departamento de Matemáticas, University of Almeria, E-04071, Almeria, Spain

E-mail address: mizurdia@ual.es 